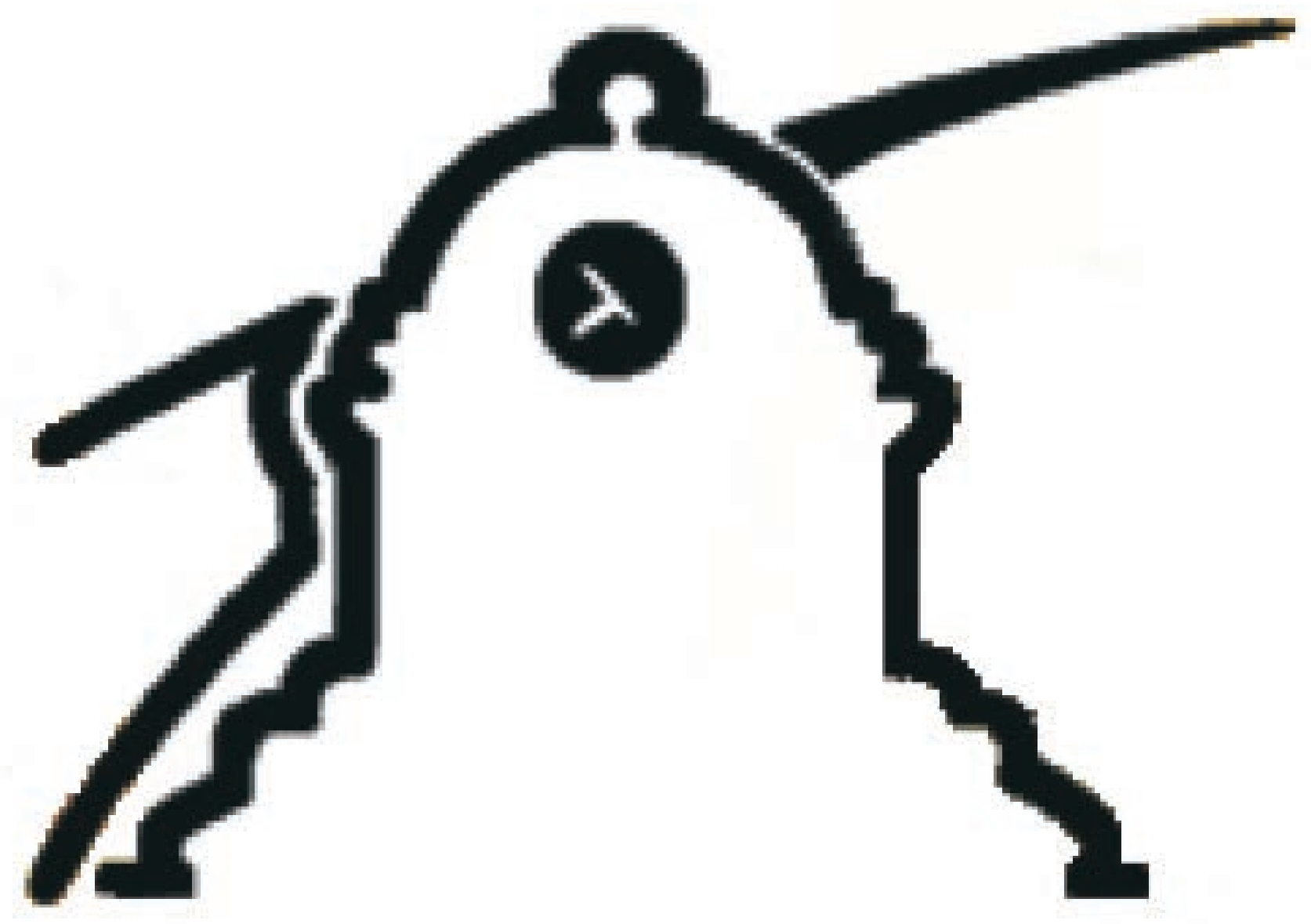

INVESTIGACIÓN UAM 



\title{
LOS OBJETIVOS DEL MILENIO Y EL DESARROLLO SOSTENIBLE.
}

\author{
MILLENIUM DEVELOPMENT GOALS AND \\ SUSTAINABLE DEVELOPMENT
}

Jahir Rodríguez Rodríguez ${ }^{1}$

Palabras claves: Objetivos del Milenio, pobreza, desarrollo sostenible. Urbanización.

Keywords: Millenium Development Goals, poverty, sustainable development. urbanization.

\section{Resumen.}

La creciente globalización que nos ha tocado vivir posee, como la mayoría de los fenómenos económicos y sociales, consecuencias positivas por un lado y negativas por otro. En este contexto se sitúa la actuación de las instituciones multilaterales de desarrollo quienes están adquiriendo mayor protagonismo en nuestros días.

De las múltiples iniciativas emprendidas por estas instituciones, teniendo como germen la Cumbre del Milenio y la Cumbre Mundial de Desarrollo Sostenible, destacan los Objetivos de Desarrollo del Milenio que constituyen una ambiciosa agenda para la resolución de los principales y más graves problemas de la humanidad.

Las reflexiones que se presentan están asociadas a los Objetivos del Milenio y sus relaciones con el desarrollo sostenible y el desarrollo urbano y revisar la evolución de estos Objetivos y cuáles son las principales carencias o necesidades de cara a su consecución en el plazo previsto del año 2015.

\section{Abstract}

The increasing globalization trend we live in, as most economic and social phenomena, has positive and negative consequences. In this context, the actions of multilateral
1 Magíster en Ciencias Políticas. Magíster en Educación. Planificador urbano. Educador popular. Coordinador de la Maestría en Desarrollo Regional y Planificación del Territorio; Director de la Revista ANFORA, Universidad Autónoma de Manizales.

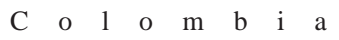 Jrodriguez17@telmex.net.co

\section{A N Universidad Autónoma de Manizales


development institutions, which have been getting more protagonism these days, are focused.

Multiple initiatives have been undertaken by these institutions, brought about in the Millenium Summit and the Sustainable Development World Summit, the Millenium Development Goals are highly important because of its ambitious agenda for the solution to the main and most serious problems of humankind.

Our reflections are based on the Millenium Development Goals and their relation to sustainable development and urban development. Also, we review the evolution of these goals and the main deficiencies or needs in terms of their completion before their meeting point set out for the year $2015 .^{2}$

\section{Palabras iniciales.}

La Cumbre Mundial sobre Desarrollo Sostenible celebrada en septiembre de 2002 en la ciudad sudafricana de Johannesburgo, auspiciada por las Naciones Unidas (NN.UU), fue concebida como continuación de la Cumbre de la Tierra de Río de Janeiro de 1992. Su objetivo principal era el de dar respuesta de forma más realista a las cuestiones planteadas (pero no solucionadas) de la década anterior, que se habían manifestado por primera vez en la Conferencia sobre Medio Ambiente Humano de Estocolmo de la década de los setenta.

Diferentes reuniones multilaterales, tanto de las NNUU como de otros organismos tales como la Organización Mundial del Comercio (OMC) o el Banco Mundial (BM), incidieron en el hecho de que, a pesar de las buenas intenciones de la Cumbre de Río, su planteamiento adolecía de una serie de errores que la hacían prácticamente inviable. En concreto, la Declaración del Milenio de las NNUU (septiembre 2000) así como la Agenda 21 (1992) expresan de forma suficientemente explícita la necesidad de reconducir o reformular los principios que guiaron la Cumbre de la Tierra.

El desarrollo sostenible es definido por las Naciones Unidas (2000) como "el desarrollo que satisface las necesidades del presente sin comprometer la capacidad de generaciones futuras para satisfacer las suyas propias." De forma específica se recoge la necesidad de plantear el aspecto social del desarrollo económico y, a su vez, éste hacerlo compatible con los objetivos medioambientales. Es decir, tal y como establecen las Naciones Unidas (2005): “las políticas de desarrollo tendrán que estar orientadas a proteger nuestros bosques, nuestros mares y nuestra fauna, así como a mejorar su productividad si queremos que los pobres puedan cerrar la brecha de desigualdad abierta en los últimos cincuenta años".

Así pues, el foco de atención pasa a ser también la sociedad y no sólo la naturaleza, de modo que el eje "persona - entorno" adquiere prioridad en el planteamiento económico. De esta forma se da respuesta a lo que muchos analistas achacaban de que la preocupación por el medioambiente descuidaba en cierto modo al individuo como agente social, ya que si bien es cierto que desacertadas políticas y deficientes gestiones públicas contribuyeron a desastres ambientales, también es verdad que éstas fueron causa de la creciente desigualdad de ingresos e, incluso, de revueltas sociales.

En la Cumbre del Milenio de las Naciones Unidas celebrada en Nueva York en el año 2000, los Estados miembros de las Naciones Unidas reafirmaron su compromiso de
2 Resúmenes traducidos por el gestor de traducciones de Instituto de Idiomas de la UAM, Carlos A. Muñoz Torres, Máster en Teoría de la Traducción, Universitat Autónoma de B arcelona. Resúmenes revisados por Kevin Guzzo, nativo del inglés, estudiante de la Maestría en Traducción de la Universidad Autónoma de Manizales.

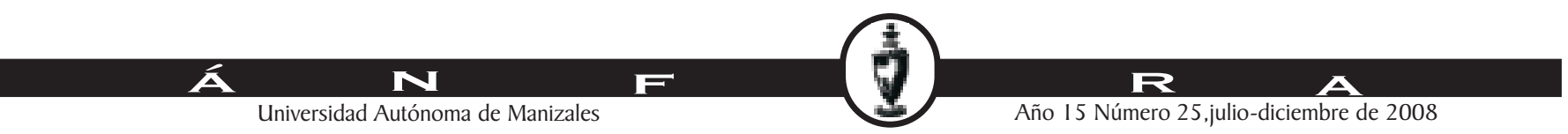


luchar por un mundo en el que el desarrollo sostenible y la eliminación de la pobreza tuvieran máxima prioridad. Fue esta una oportunidad histórica de convenir en un proceso para la revisión fundamental del papel de esta institución y de los desafíos a los que enfrenta en el nuevo siglo y en este sentido, como consecuencia principal la Cumbre se encuentra la conocida como Declaración del Milenio en el que se plasman los Objetivos de Desarrollo del Milenio.

Éstos surgen como derivación de acuerdos e iniciativas emprendidas por diferentes conferencias mundiales organizadas por las Naciones Unidas en los últimos años. Los Objetivos han sido aceptados, con carácter global, como marco para la medición de los progresos en materia de desarrollo y para ello centran la labor de la comunidad mundial en la introducción de mejoras importantes y mensurables en la vida de las personas. De ellos, cabe destacar el establecimiento de criterios para cuantificar los resultados, ya no sólo de los países en desarrollo, sino también de los países desarrollados que contribuyen a financiar programas de cooperación, así como de las instituciones multilaterales que ayudan a los países destinatarios a aplicarlos.

\section{La cumbre mundial sobre desarrollo sostenible}

La Cumbre de J ohannesburgo nace ya desde el mismo momento de su partida con un doble desafío: en primer lugar mejorar las condiciones de vida de todo ser humano y, por otro lado, proteger el medioambiente. Es el primero de estos retos el que supone una novedad en el planteamiento interno de las NNUU y al que se le presta atención en este apartado, sin olvidar que todo este movimiento tiene su origen en el constante y creciente deterioro medioambiental.

Todo el proceso preparatorio de la Cumbre, así como otras conferencias relacionadas tales como la Conferencia Ministerial de Doha (noviembre de 2001) y la Conferencia Internacional sobre Financiamiento al Desarrollo de Monterrey (marzo de 2002) tienen como objetivo la búsqueda de un balance entre el desarrollo económico - social y medioambiental. Más aún, estos tres aspectos interdependientes se plantean como los componentes principales del denominado "desarrollo sostenible", expresión que da nombre a la Cumbre y que pretende recoger los aspectos comentados anteriormente.

De forma específica dentro de los objetivos y requisitos necesarios de esta nueva concepción del desarrollo socioeconómico planteado en J ohannesburgo aparece junto a la protección del medio natural aspectos tales como la erradicación de la pobreza o el cambio en los patrones de consumo y de producción. Se muestra de esta manera la preocupación internacional por la prosperidad, la seguridad y la estabilidad del mundo y se hace hincapié en la necesidad de reducir las diferencias. Echando la vista atrás frente a los planteamientos exclusivamente ecológicos de la Cumbre de Estocolmo, puede llamar la atención el hecho de que, por ejemplo, la protección de la biodiversidad se sitúe de modo paralelo a la disminución de las diferencias entre los pobres y no pobres de la Tierra. J unto a ello destaca la circunstancia de que "por primera vez en la historia de la humanidad, la sociedad tiene la capacidad, el conocimiento y los recursos para erradicar la pobreza y el subdesarrollo" (Mejía, H, 2002).

En cuanto a los objetivos propios establecidos conviene recordar la necesidad del uso inteligente de los mismos. Así tomando la referencia de las Declaración de Naciones Unidas (2000): "estos no pueden capturar la totalidad y complejidad de las transferencias individuales y colectivas necesarias para el desarrollo sostenible". Es

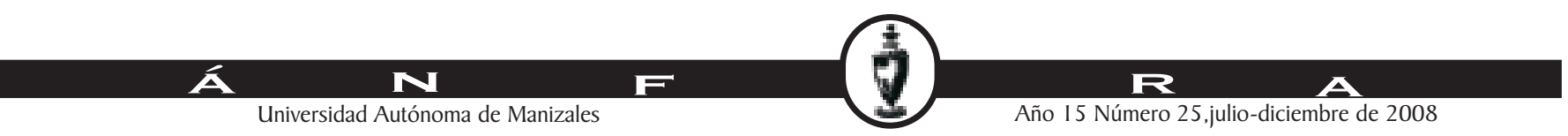


decir, se hace necesaria la adaptación de los objetivos al entorno local al que se aplica y a la realidad socioeconómica del mismo.

Asimismo, es necesario señalar que esta Cumbre pretende reflejar las nuevas características de la problemática del desarrollo socioeconómico. En concreto, la tan repetida globalización que vivimos en la actualidad es foco de atención en las discusiones y conclusiones.

Ciertamente el fenómeno de la globalización ha añadido una dimensión importante al desarrollo sostenible al abrir nuevas oportunidades incrementando los flujos financieros y materiales mediante la integración de mercados y el favorecimiento de la movilidad de capital. Sin embargo, también es necesario mencionar las amenazas latentes que conlleva esta corriente, puesto que tal y como establece J. Sachs (2002) "si los países ricos culpan a los desafortunados (aduciendo su inadecuación a la globalización) no sólo crearemos bolsas de pobreza más profundas sino también un descontento creciente"

De igual modo, la preocupación por la dignidad humana es recogida en la Declaración de J ohannesburgo expresando una serie de requisitos necesarios entre los que se sitúa el acceso a recursos financieros, la apertura de mercados, el uso y transferencia de tecnología y la importancia de la educación y el aprendizaje. No obstante, por encima de ellos y como requisito indispensable para el desarrollo sostenible en todos los documentos y declaraciones multinacionales sobre este tema aparece la erradicación de la pobreza.

Asi mismo, esta Cumbre tiene como uno de principales objetivos los ya anticipados por las NNUU en la Declaración del Milenio, que se pretenden conseguir antes del año 2015.

\section{El desarrollo como derecho humano.}

Hoy por hoy, podemos defender la tesis de la vigencia, aunque limitada, y la plena legitimidad del derecho al desarrollo humano y sostenible sobre la base de textos jurídicos internacionales elaborados principalmente en el marco de las Naciones Unidas, por un lado, y, por otro, con base en la cultura y la filosofía de los derechos humanos y fundamentales, y los valores con vocación universalista en que se inspiran. Asimismo, debe tenerse en cuenta la satisfacción de las necesidades básicas y humanas, como la alimentación, el agua potable, la vivienda, la salud o la educación, aunque el modo de satisfacerlas pueda variar según el contexto histórico y cultural en el que los distintos grupos e individuos se desarrollen. La satisfacción de estas necesidades es condición ineludible para que todo individuo pueda estar en condiciones de ejercer y disfrutar de todos los derechos humanos y libertades fundamentales.

De este modo, todo individuo debe estar en condiciones de poder desarrollar al máximo sus capacidades y sentirse libre y digno de sí mismo. Por esta razón, la meta final consiste en lograr el máximo bienestar humano y hacer realidad la dignidad para todos, sin discriminación, y no sólo para unos pocos privilegiados. En este sentido, el desarrollo es un derecho humano que engloba el conjunto de los derechos humanos y pone de manifiesto la universalidad, interdependencia e indivisibilidad de dichos derechos, tal y como afirma la Declaración y Programa de Acción de Viena de 1993, fruto de la Conferencia Mundial de Derechos Humanos celebrada en dicha ciudad. Esta interpretación está en consonancia con lo dispuesto en los preámbulos de los Pactos

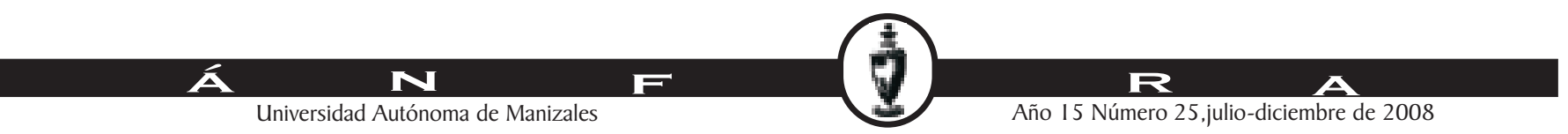


Internacionales de los Derechos Económicos, Sociales y Culturales y de los Derechos Civiles y Políticos, adoptados en el marco de las Naciones Unidas en 1966, cuando se afirma lo siguiente: "que, con arreglo a la Declaración Universal de Derechos Humanos, no puede realizarse el ideal del ser humano libre, en el disfrute de las libertades civiles y políticas y liberado del temor y de la miseria, a menos que se creen condiciones que permitan a cada persona gozar de sus derechos civiles y políticos, tanto como de sus derechos económicos, sociales y culturales". (Naciones Unidas: 1966).

Entre los objetivos de un derecho al desarrollo así concebido figura el mejorar el bienestar, la dignidad y la calidad de vida de todos los seres humanos, así como lograr una mayor igualdad económica y social, atendiendo especialmente a las necesidades de los individuos y grupos más vulnerables, y respetando la diversidad cultural. Para ello, debe erradicarse la pobreza, en tanto que objetivo primordial, y todos los Estados deben ratificar todos los tratados relativos a los derechos humanos sin reservas. Asimismo, los poderes públicos y privados deben ser controlables y fiscalizables por medios democráticos y deben rendir cuentas de sus actividades de manera transparente, de modo que pueda verificarse si se han ajustado o no a la ley, facilitando así la lucha contra la corrupción y la criminalidad.

También puede considerarse como objetivo del desarrollo el logro de una paz justa en la que se respeten y protejan todos los derechos humanos para todos, pues de lo contrario sería una paz aparente que oculta una situación de opresión y violencia ejercida desde los poderes dominantes. Ésta es la mejor garantía de la seguridad para todos, a la cual debe unirse un desarme progresivo que disminuya la capacidad de amenazar y destruir, y que libere recursos económicos y humanos para actividades pacíficas. Además, el desarrollo debe ser sostenible, es decir, las generaciones futuras deberían heredar y disfrutar de nuestro planeta en las mismas condiciones que las generaciones presentes y, si es posible, aún mejor y más equitativamente.

\section{Los obstáculos al desarrollo.}

Las fuerzas y poderes hegemónicos presentan la actual globalización como un fenómeno irresistible, irreversible y, lo que es peor, sin posible alternativa, como si se tratara de una verdad revelada e indiscutible. Por ello, pretenden justificar en nombre de tal globalización políticas económicas denominadas de "austeridad" o de "ajuste estructural" en los países del tercer mundo, las cuales contribuyen a perpetuar un sistema de dominación económica y política mediante un intercambio económico y comercial enormemente desequilibrado, desigual e injusto. Son los denominados "programas de ajuste estructural" promovidos por las instituciones financieras internacionales, como el Fondo Monetario Internacional (FMI) y el Banco Mundial (BM), e impulsados por los Estados de los países más ricos e industrializados y las empresas transnacionales.

Dichos programas de ajuste estructural, rebautizados con el nombre de "estrategias de lucha contra la pobreza", confían en que el mero crecimiento económico aportará por sí solo el desarrollo. Dicho crecimiento económico, si se da tal y como lo proponen las instituciones financieras internacionales mencionadas, beneficia sobre todo a las capas más privilegiadas de la sociedad y aumenta aún más la dependencia de los países del tercer mundo, lo cual se refleja, por ejemplo, en el aumento continuo de la deuda externa de estos países.

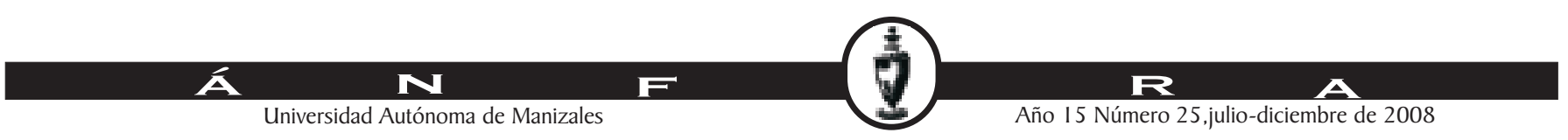


Los intereses por el pago de esta deuda constituyen la base del paradójico fenómeno consistente en que el flujo de capitales es, hoy en día, predominantemente del "Sur" o "Periferia", cada vez más pobre y endeudado, hacia el "Norte" o "Centro", rico e industrializado, y no al revés, como debería suceder si se quiere invertir este proceso de pauperización global en que está degenerando la actual mundialización del mercado.

A ello se añade que la Ayuda Oficial al Desarrollo (AOD) de los Estados de los países más ricos e industrializados hacia los países del tercer mundo no sólo no aumenta, sino que disminuye. Y, si bien en esta ayuda oficial no todo es "trigo limpio", las corrientes de capital privado no han logrado ni mucho menos suplir esta tendencia decreciente no sólo en cuanto a la cantidad, sino también en cuanto a la "calidad" de la ayuda. El capital privado, por su propia naturaleza, se inclina más por la mera rentabilidad económica y la obtención de beneficios a corto plazo, así como por la garantía de que estos beneficios puedan ser repatriados por las instituciones financieras y bancarias de donde procede dicho capital privado, en vez de reinvertirse en los lugares donde se han obtenido tales beneficios, y favorecer así su desarrollo. Todos estos aspectos deben ser considerados como obstáculos a la realización del derecho al desarrollo.

Todo esto pone de relieve que los derechos humanos y el desarrollo humano y sostenible deben realizarse en un mundo cada vez más mercantilizado, es decir, donde el comercio ocupa un lugar primordial. Comercio y desarrollo son actividades que deben compatibilizarse, pero teniendo en cuenta que el comercio es un instrumento y el desarrollo y los derechos humanos el fin, y no al revés.

Frente a lo que afirma e impone la Organización Mundial del Comercio, es el comercio el que debe subordinarse a los individuos y a los grupos humanos: los seres humanos e incluso muchos recursos naturales no son reductibles a mera mercancía vendible en el mercado mediante un precio. Además, los beneficios de la actividad comercial deberían ser equitativamente repartidos entre todos los individuos y grupos humanos, atendiendo especialmente las necesidades de los individuos y grupos más vulnerables.

En definitiva, la idea que debe orientar este enfoque debe ser clara: tras la concepción de un derecho al desarrollo humano y sostenible como derecho humano figura la aspiración de que la libertad y la dignidad deben estar al alcance de todos los seres humanos y no sólo de unos pocos privilegiados. Además, debe ser realizable, es decir, deben crearse las condiciones para que todos puedan satisfacer sus necesidades legítimas y realizar sus proyectos o planes de vida igualmente legítimos. Asimismo, deben tenerse en cuenta no sólo las generaciones presentes, sino también las futuras.

\section{Objetivos del Milenio: Los desafíos urbanos del desarrollo.}

En los países en vías de desarrollo, cerca de dos mil millones de personas viven actualmente en medios urbanos. De acuerdo a las previsiones de las Naciones Unidas, esta cifra se duplicaría en los próximos 30 años. Hoy, se estima que un tercio de estos habitantes urbanos, pobres, se alojan en países pobres.

El programa de las Naciones Unidas para los Asentamientos Humanos (ONU-Hábitat) tiene la responsabilidad de apoyar a los Estados miembros para cumplir una de las principales metas urbanas de los objetivos del Milenio para el Desarrollo: de aquí a

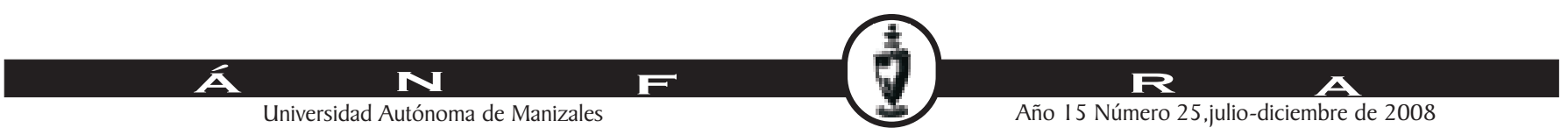


2020, mejorar sensiblemente la vida de por lo menos 100 millones de habitantes de las ciudades de emergencia (objetivo 7: "Asegurar un desarrollo sostenible “).

La intención es modesta frente a los desafíos de la crisis urbana mundial, porque sólo tiende a favorecer del 10 al 15\% de las poblaciones citadinas que viven en situación de extrema precariedad.

Sin embargo, la misma merece ser destacada porque reconoce un desafío mayor del desarrollo para la comunidad internacional: las ciudades pobres.

Si este objetivo de mejorar las condiciones de vida de los pobres en las ciudades de emergencia trata de remediar las carencias observadas hace décadas en medios urbanos, no es la única iniciativa que podría garantizar un impacto positivo en las ciudades en desarrollo y en las familias que las habitan.

Recordemos otros objetivos del Milenio: disminuir a la mitad, de aquí a 2015 el número de personas que no tienen acceso al agua potable ni al saneamiento, integrar los principios de desarrollo sostenible en las políticas nacionales e invertir la tendencia actual en cuanto a la pérdida de los recursos medioambientales.

Contra la pobreza extrema y el hambre, reducir a la mitad la proporción de individuos cuyos ingresos sean inferiores a un dólar por día de aquí a 2015.

En el plano educativo, todos los niños deberían concluir un ciclo completo de educación primaria. En el campo sanitario, la mortalidad infantil se reduciría dos tercios, y la tasa de mortalidad maternal se bajaría tres cuartas partes, la propagación del HIV/sida se detendría y se revertiría la tendencia actual. Todas estas medidas, si fructifican, se orientan tanto a las poblaciones rurales como a las urbanas.

\section{Urbanización de la pobreza.}

En los países más ricos, menos del $16 \%$ de todas las familias de las zonas urbanas vive en la pobreza. Pero en las zonas urbanas de los países en desarrollo, el $36 \%$ de todas las familias y el $41 \%$ de todos los hogares a cargo de una mujer viven con ingresos que están por debajo del umbral de pobreza definido a escala local. La urbanización y la feminización de la pobreza han traído como resultado que más de 1.000 millones de pobres vivan en zonas urbanas sin viviendas adecuadas ni acceso a los servicios básicos. (ONU-HABITAT, 2001).

Frente a esta comprobación preocupante se plantea la cuestión de los medios y de los enfoques. Los recursos financieros de la ayuda pública internacional se estancan. Estos recursos llegaban a 60.000 millones de dólares US en 1992, fueron disminuyendo progresivamente para aumentar a ese mismo monto en 2003. Esta ausencia de medios suplementarios no es el único tema de inquietud. I gualmente hay que cuestionarse en lo que respecta a los enfoques y a los métodos adoptados para mejorar la situación de los habitantes urbanos pobres.

\section{¿Qué alternativas para mejorar las condiciones de vida en las ciudades pobres?.}

Para el Instituto Internacional para el Medio Ambiente y el Desarrollo, es importante

3 Término acuñado por ONU-

Hábitat en los 90

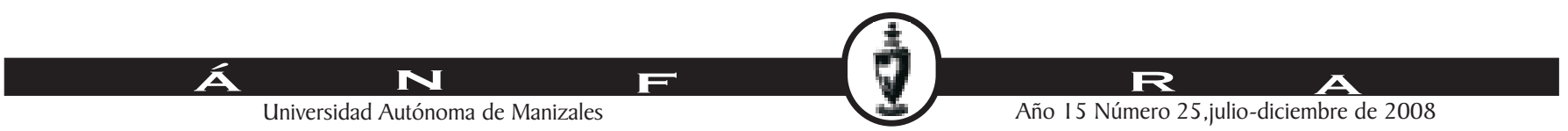


darle prioridad a la acción y promover las vías alternativas que existen hoy, movilizando verdaderamente y de forma diferente a los actores implicados en los niveles nacionales y sobre todo, locales.

Si bien los pobres son los principales beneficiarios de los objetivos del desarrollo, muy raramente se los consulta y no se los integra al tomar las decisiones. En lugar de verlos como "el problema", se los debería considerar como verdaderos actores y miembros activos de una asociación, luchando contra la pobreza. Sus competencias y sus aptitudes se conocen mal y por eso, rara vez se aprovechan.

Los gobiernos locales juegan un rol primordial en toda estrategia que apunte a mejorar las condiciones de vida urbana.

Son ellos quienes, para bien o para mal, van a decidir las políticas inmobiliarias y las reglas de otorgamiento de terrenos a las familias pobres. Son ellos los que decidirán entre el desalojo de las ciudades de emergencia o su rehabilitación. Son ellos, también, quienes les darán participación o no a las asociaciones comunitarias en cuanto a las tomas de decisiones relativas a estos barrios pobres. Las ONGs locales o internacionales tienen igualmente un rol que deben hacer valer en cuanto al apoyo que aporten a estas asociaciones de base y con respecto al fortalecimiento de sus capacidades para negociar con las autoridades urbanas.

Nuevas reglas de juego en materia de gestión urbana. Dentro de una perspectiva de planificación participativa, es importante, tanto para las autoridades como para las organizaciones de apoyo, que los habitantes se organicen y se estructuren con el fin de estar preparados para dialogar sobre bases sólidas con los actores públicos y privados. Esto es particularmente acertado en los ámbitos claves de la rehabilitación urbana. Por ejemplo para el abastecimiento de agua potable y el saneamiento en los barrios de hábitat precario, las poblaciones implicadas deben estar asociadas a la planificación y a las decisiones y participar en las realizaciones por medio de su trabajo y con una contribución financiera.

Otra cuestión prioritaria reside en la regularización de la propiedad de los terrenos de las ciudades. Estos terrenos expuestos a la presión comercial pueden transformarse rápidamente en la presa de los inversores inmobiliarios. Se trata de oponerse a esta tendencia que se observa a menudo en ámbitos urbanos y de favorecer el derecho a la tierra de los ocupantes, facilitando los procesos administrativos y reduciendo el costo de los trámites emprendidos. La seguridad en cuanto a la propiedad del suelo tendrá un efecto muy positivo sobre las inversiones realizadas por los habitantes para mejorar sus viviendas y las infraestructuras vecinales. Como es sabido, los pobres construyen la mayoría de las veces, sus viviendas progresivamente en el transcurso de los años y generalmente, fuera de toda legalidad. Un mejoramiento de la calidad de la construcción pasa también por una adaptación de los sistemas financieros que, muy a menudo, no benefician a las personas que no tienen salarios fijos y que no poseen títulos de propiedad. Sería necesario alentar cambios al respecto. En efecto, numerosos ejemplos demuestran que los préstamos acordados a las familias de bajos ingresos son rentables y estables cuando están asegurados por instituciones que se esfuerzan en responder a las necesidades de los pobres, a sus capacidades financieras y a sus modos de vida. Obteniendo garantías que no sean individuales, hipotecarias o inmobiliarias por parte de las familias pobres, en las estructuras de crédito abiertas a esta clientela se comprueba que los pagos se efectúan en el plazo requerido, a pesar de

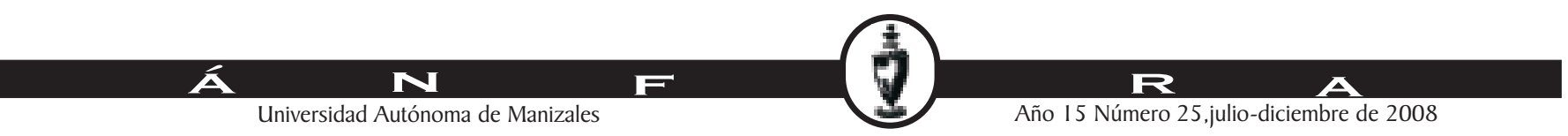


las tasas de interés a menudo elevadas. Esto se verifica en tanto y en cuanto los deudores puedan organizarse colectivamente y posean los consejos necesarios.

\section{Los objetivos urbanos del Milenio, más que una cuestión material y financiera.}

La revalorización de las ciudades de emergencia abarcaría a 100 millones de individuos en los próximos quince años, según los objetivos de desarrollo identificados por las Naciones Unidas para el Milenio. Cuando la población urbana se acrecienta en los países en desarrollo, alcanzar esta meta no pasa únicamente por una rehabilitación material de las condiciones de existencia de los pobres.

Pasa también y ante todo, por una rehabilitación moral que otorgue confianza y respeto a los pobres. La misma se concretará a través de relaciones más transparentes y responsables tanto de parte de las autoridades públicas locales y nacionales, como de parte de otros actores urbanos, privados y ONGs, en una asociación eficaz que apunte a alcanzar los objetivos urbanos de desarrollo del milenio, fructíferos para todas las partes comprometidas en este desafío.

\section{Evolución de los objetivos.}

El progreso en la consecución de estos Objetivos no tiene un signo bien definido. Así, algunos países están bien encaminados cara a su consecución mientras que en otros (más de los que sería deseable) es probable que no se puedan conseguir si se prosigue con la actual evolución de progreso mundial. Esto es debido a muchas razones, entre las que muchas veces se encuentran el gasto público insuficiente e ineficiente, las cargas abrumadoras de la deuda, el acceso inadecuado a los mercados en los países desarrollados y la disminución de la asistencia oficial para el desarrollo.

Situados en un momento crucial de la evolución de los objetivos, al haber transcurrido ya diez años de la aprobación del Milenio y a menos de cinco años de que se cumpla el plazo para su cumplimiento se está en condiciones de efectuar una evaluación de los mismos. Ésta constituye también una tarea realizada por las propias NNUU a través de Informes Anuales y el trabajo de otras instituciones multilaterales y bilaterales tales como el FMI mediante respectivos I nformes de Seguimiento que se llevan a cabo con periodicidad regular y de manera global, entre los que destacan los Indicadores de los objetivos de desarrollo del milenio.

De hecho, las principales conclusiones que pueden ser extraídas para el año 2005 (NNUU, 2005) se recogen a continuación clasificadas según cada objetivo:

Por lo que se refiere al Objetivo 1 (Erradicar la pobreza extrema y el hambre) se constata que las tasas mundiales de pobreza se están reduciendo, principalmente en el continente asiático. Por lado contrario nos encontramos la situación del África subsahariana, donde millones de personas más han caído en la pobreza extrema y, por tanto, donde los pobres son cada vez más pobres.

En este sentido se han logrado progresos en la lucha contra el hambre, pero en algunas regiones ha habido un retroceso debido a factores tales como el lento aumento de la producción agrícola o a la evolución demográfica. Desde el año 1990, hay varios millones más de personas que sufren hambre crónica en el África subsahariana y Asia meridional, donde la mitad de los niños menores de 5 años padecen de malnutrición.

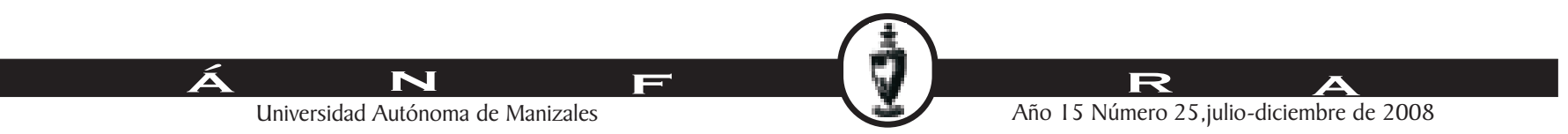


De modo similar el Objetivo 2 (Lograr la enseñanza primaria universal) observa una evolución favorable con alguna marcada excepción. Concretamente cinco regiones en desarrollo se están acercando a una tasa de matriculación del 100\%, mientras que en el África subsahariana, menos de las dos terceras partes de los niños están matriculados en escuelas primarias.

Evolución lenta aunque positiva también se da en el Objetivo 3 (Promover la igualdad entre los géneros y la autonomía de la mujer). Concretamente sobre este particular hay que mencionar que se están reduciendo, si bien más lentamente de lo que sería deseable, la diferencia entre géneros en la tasa de matriculación en educación primaria del mundo en desarrollo. Esto constituye un primer paso para reducir las desigualdades existentes desde hace mucho tiempo entre mujeres y hombres.

En casi todas las regiones en desarrollo, las mujeres representan una proporción más pequeña de los empleados asalariados que los hombres y con frecuencia se ven relegadas a trabajos inestables y mal remunerados. Aunque se están logrando progresos significativos, las mujeres siguen sin estar representadas de forma equitativa en los niveles más altos de gobierno, pues ocupan tan sólo el $16 \%$ de los escaños de los parlamentos del mundo.

La reducción de la mortalidad infantil recogida en el Objetivo 4 dirigida a los niños menores de cinco años supone otro ejemplo de evolución positiva pero no al ritmo necesario. Así cada año, y motivado por enfermedades que se pueden prevenir o tratar, mueren once millones de niños menores de cinco años, lo cual supone unos 30.000 al día. Esto resulta especialmente grave si se considera que la mayoría de esas vidas se podrían salvar ampliando programas existentes que promueven soluciones sencillas y además de bajo costo.

El Objetivo 5 está dedicado a mejorar la salud materna y muestra como aún queda camino por andar. Cada año muere más de medio millón de mujeres durante el embarazo o el parto. Esta cifra, multiplicada por veinte, es el número de mujeres que sufren lesiones graves o casos de discapacidad.

En este sentido se han logrado algunos progresos en la reducción de la mortalidad materna en las regiones en desarrollo, pero no en los países donde es más peligroso dar a luz como son los del África subsahariana.

EI SIDA se ha convertido en la causa principal de muerte prematura en zonas como el África subsahariana, al tiempo que ocupa el cuarto lugar dentro de las causas de muerte en todo el mundo. Por este motivo constituye el Objetivo 6 dedicado a combatir el VIH/SIDA, el paludismo y otras enfermedades.

En los países de la Europa del Este y en algunas partes de Asia, el VIH se está propagando a una velocidad alarmante. Aunque los nuevos tratamientos prolongan la vida, no hay cura para el SIDA, por lo que, para cumplir este objetivo, se deben intensificar las tareas de prevención en todas las regiones del mundo.

Cada año, de manera conjunta, dos enfermedades tales como el paludismo y la tuberculosis, causan la muerte de casi tantas personas como el SIDA, además de ocasionar graves pérdidas a las economías nacionales. El $90 \%$ de las muertes por paludismo se producen en el África subsahariana, donde se están incrementando las

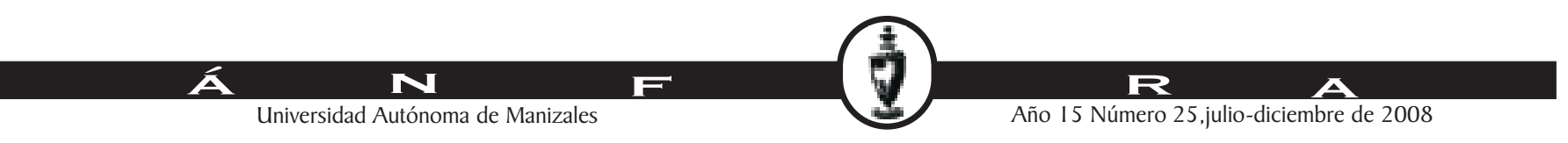


actividades de prevención y tratamiento. La tuberculosis sigue presentando una tendencia al alza, en parte como resultado del VIH/SIDA, aunque hay un nuevo protocolo internacional para detectar y tratar esta enfermedad que parece, por el momento, prometedor.

El Objetivo 7 (Garantizar la sostenibilidad del medio ambiente) también ha experimentado una evolución con luces y sombras. Así, aunque la mayoría de los países se ha comprometido a cumplir los principios del desarrollo sostenible, ello no se ha traducido en un avance suficiente para dar marcha atrás a la pérdida de los recursos ambientales del planeta.

Para alcanzar este objetivo se necesitará prestar más atención a la difícil situación de los pobres (cuya subsistencia cotidiana suele depender directamente de los recursos naturales que los rodean) y un nivel de cooperación mundial sin precedentes hasta la fecha. Las medidas adoptadas para impedir que siga deteriorándose la capa de ozono demuestran que es posible progresar.

En sentido positivo destaca que ha aumentado el acceso al agua potable, aunque una parte importante de la población mundial (aún un $50 \%$ ) sigue sin disponer de retretes u otras formas básicas de saneamiento. Casi 1.000 millones de personas viven en barrios urbanos de tugurios porque la población urbana aumenta a un ritmo muy superior al de las mejoras de viviendas y al de la disponibilidad de puestos de trabajo productivos.

La Declaración del Milenio aprobada por las Naciones Unidas representa un pacto social mundial: los países en desarrollo se esforzarán más para impulsar su propio desarrollo y los países desarrollados los apoyarán prestándoles ayuda, aliviando su deuda y brindándoles mejores oportunidades de intercambio comercial. Esto constituye la esencia del propio Objetivo 8 dedicado a fomentar una asociación mundial para el desarrollo.

En este particular los progresos alcanzados en cada uno de estos ámbitos ya han comenzado a producir resultados, pero los países desarrollados no han cumplido las metas que se habían fijado. Para cumplir los objetivos de desarrollo del Milenio, el aumento de la ayuda y el alivio de la deuda deben ir acompañados de una mayor apertura de los intercambios comerciales, de una aceleración de la transferencia de tecnología y de mayores oportunidades de empleo para el creciente número de jóvenes que viven en el mundo en desarrollo.

En resumen, la evolución de la mortalidad infantil muestra un signo favorable, al tiempo que destaca el rápido aumento de la matrícula en la escuela primaria ( 50 países han logrado la terminación universal del ciclo de educación primaria, cifra que representa un aumento respecto de los 37 países que se encontraban en esa situación en 2000). Paralelamente se constata que existen señales de que, por primera vez, están disminuyendo las tasas de infección por el VI H/SIDA en países de alta incidencia (como Haití, Uganda y Zimbabwe)

No obstante, y como principal lastre en esta evolución se observa que los avances siguen siendo desiguales. Un gran número de países, especialmente en África y América Latina, todavía no logran avances importantes en la lucha contra la pobreza, y el progreso en los indicadores de desarrollo humano en Asia meridional ha sido insuficiente.

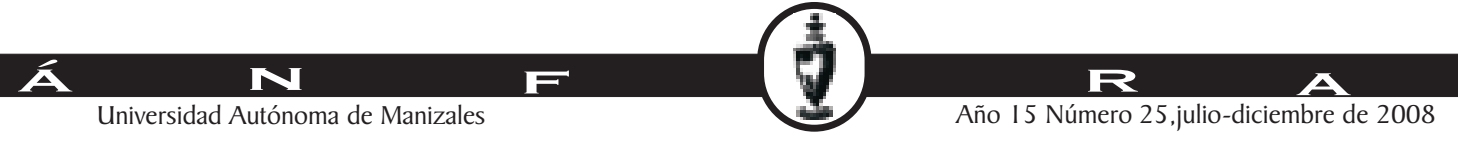




\section{Horizonte pendiente.}

Falta menos de cinco años para alcanzar los Objetivos del Milenio que la comunidad internacional fijó a finales de siglo. En 2005, se realizó la Cumbre del Milenio +5 con el objetivo de analizar su evolución. Esta Cumbre que vino precedida de una serie de audiencias y reuniones con la sociedad civil, las ONGs y el sector privado, reafirmó su compromiso con respecto a la responsabilidad mutua para lograr resultados y centró la atención en aumentar los recursos de ayuda.

En esta línea, la Declaración de París sobre Eficacia de la Ayuda al Desarrollo, que reunió en marzo de 2005 a responsables de instituciones multilaterales de desarrollo, entre las que destacan el Banco Mundial o el Fondo Monetario I nternacional, sirvió para que los donantes reforzaran los compromisos de incrementar la eficacia de la ayuda por medio de una mejor armonización y mayor convergencia. I gualmente en la reunión anual del G-8 llevada a cabo el año pasado en la Cumbre de Gleneagles sirvió para formular nuevos compromisos de ayuda y alivio de la deuda. Los países en desarrollo reafirmaron su compromiso de fortalecer la gobernabilidad y aplicar enérgicas estrategias de desarrollo.

Sin embargo, y pese a lo dicho y alto grado de concienciación e implicación en determinados niveles, se puede afirmar que la consecución de los Objetivos del Milenio está lejos de ser alcanzada. Muchos países, sobre todo de África y Asia meridional, no han progresado conforme a lo previsto y, así, nos encontramos con numerosos ejemplos de la lentitud o el fracaso de los esfuerzos. Ello viene explicado por la insuficiencia de los recursos y una deficiente gestión en determinados niveles gubernamentales que hacen, como fue ya apuntado, que más de diez millones de niños mueran anualmente a causa de enfermedades fácilmente prevenibles o que en muchos países en vías de desarrollo solamente dos tercios de los hogares de ingreso bajo de las zonas urbanas y un cuarto de los de las zonas rurales tengan acceso a algún tipo de red de saneamiento.

I gualmente, sigue siendo frecuente que la ayuda no se destine a los verdaderos necesitados y que las instituciones financieras internacionales continúen poniendo énfasis en la concesión de créditos y préstamos, así como en la preparación de informes, y no en los resultados en términos de desarrollo. En definitiva hace falta intensificar los esfuerzos para implementar la acción mundial y la responsabilidad mutua por la obtención de resultados forjada en las Cumbres señaladas. Asimismo, la actuación de modo global y no tanto local sigue siendo una necesidad más que una realidad en este tipo de propuestas y actuaciones, las cuales deben ir acompañadas de medidas que permitan utilizar la ayuda de manera más eficaz en la procura de incrementar las responsabilidades mutuas.

En este sentido, y tal y como se señala en los últimos informes sobre seguimiento mundial, un elemento clave en este sentido es mejorar la gobernabilidad, tanto en los países en desarrollo como en el resto del mundo, a fin de intensificar la rendición de cuentas con respecto a la utilización de los recursos y los resultados en términos de desarrollo. Así pues, se debe prestar atención de manera especial en la medición y el seguimiento de la gobernabilidad, en apoyo de una rendición de cuentas más intensa y mejores resultados con respecto a los obj etivos propuestos.

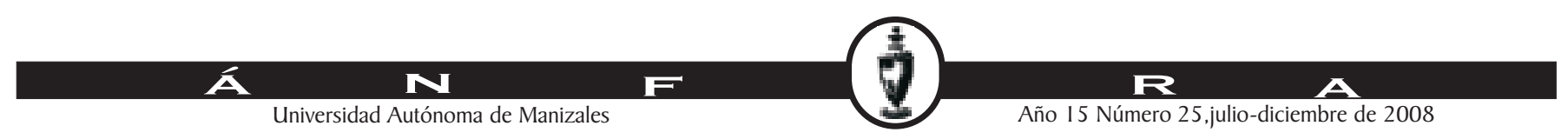


La evaluación y control de la gobernabilidad debería ayudar a definir mejor las opciones para aumentar la asistencia ofrecida, y apoyar las iniciativas globales para aumentar la transparencia y la rendición de cuentas (tanto a nivel nacional como mundial). Con esta finalidad recientemente el Fondo Monetario Internacional y el Banco Mundial a través de su Comité para el Desarrollo han apuntado una serie de medidas encaminadas a intensificar las responsabilidades mutuas.

En concreto se destacan seis medidas fundamentales que, además de servir para fortalecer las responsabilidades mutuas, servirían también para acelerar los progresos en la consecución de los Objetivos del Milenio. Estas medidas son las siguientes:

Medida 1: El crecimiento ha ayudado a reducir la pobreza, pero para acelerar y uniformar los progresos hace falta mejorar la infraestructura y el clima para la inversión en los países.

Medida 2: Los progresos realizados recientemente con respecto al desarrollo humano señalan la necesidad de contar con ayuda más flexible y de mejorar la coordinación y la gobernabilidad.

Medida 3: En 2005 se hicieron importantes compromisos relativos al suministro de ayuda y de alivio de la deuda, pero es preciso realizar un seguimiento atento a fin de protegerse de los riesgos que podrían obstaculizar su eficaz implementación. Así mismo, hace falta revitalizar las reformas comerciales.

Medida 4: La atención de las instituciones financieras internacionales debe dejar de ponerse en la gestión de los insumos y concentrarse en el logro de resultados concretos, lo que plantea grandes desafíos tanto para los organismos internacionales como para los países en vías de desarrollo.

Medida 5: Se debería hacer un seguimiento permanente de la gobernabilidad a fin de ayudar a evaluar los progresos, mejorar la rendición de cuentas y exigir mayores consecuciones.

Medida 6: La comunidad internacional debe apoyar los esfuerzos orientados a fortalecer los sistemas de gobernabilidad ratificando y apoyando los sistemas internacionales de frenos y contrapesos.

J unto a estas seis medidas para poder cumplir los compromisos de ayuda y alivio de la deuda que posibilitaría la consecución de los Objetivos es necesario intensificar determinadas actuaciones que se vienen emprendiendo de modo global. Así, de manera específica cabría hablar de la necesidad de asegurar el alivio de la deuda mediante un cuidadoso establecimiento de puntos de referencia y seguimiento de los flujos de ayuda.

Además, en línea con lo establecido previamente respecto a la ayuda oficial para el desarrollo, resulta imprescindible hacer un seguimiento de sus desembolsos y de su composición para así determinar las mejoras en la calidad y las modalidades de la ayuda. Concretamente las estrategias deberían encaminarse a la procura de una ayuda menos volátil, más flexible, y en consonancia con las estrategias para la reducción de la pobreza). Finalmente, también se sostiene que en los países receptores de este tipo de ayudas se debe mejorar de manera notable el seguimiento del gasto público y de la gestión de sus finanzas públicas.

\section{A N} Universidad Autónoma de Manizales 
En definitiva, con respecto a la evolución reciente en la consecución de los Objetivos del Milenio "estamos logrando progresos en muchos países, y ello demuestra que los esfuerzos en pos del desarrollo pueden generar resultados. No obstante, faltando sólo un decenio para alcanzar los objetivos, urge que tanto los países en desarrollo como la comunidad de donantes mejoren la gobernabilidad para que podamos obtener los resultados previstos" (Naciones Unidas, 2005).

\section{Palabras finales.}

La valoración y evaluación del desarrollo de los Objetivos del Milenio admite, como es habitual en estos casos, múltiples interpretaciones. No obstante, el punto de partida debería descansar en la trascendencia del esfuerzo realizado por la comunidad internacional en situar estos Objetivos como pilar fundamental de la actividad de los organismos de cooperación internacional y de los propios gobiernos de los países desarrollados. Al margen de otras consideraciones, lo que sí resulta un hecho evidente es que en la actualidad se cuenta con unas metas perfectamente especificadas y, por ende, cuantificables en cuanto a su evolución y consecución, lo cual no se había dado de esta manera hasta el momento actual.

A partir de aquí se abre un abanico de consideraciones que van desde un gran optimismo hasta la decepción total según sea de quien proceda y en función de la parte que está implicada. Una de las principales llamadas de atención, sino la principal, sobre los riesgos de no alcanzar los Objetivos viene dado por el conocido como I nforme Sachs (2005) que fue entregado al Secretario General de la ONU relatando e informando sobre las mejores estrategias para lograr los Objetivos de Desarrollo del Milenio.

Las recomendaciones de este I nforme agrupadas en torno a diez medidas centran sus implicaciones en dos aspectos clave para el sistema internacional de cooperación al desarrollo. Concretamente, se habla del reparto geográfico de la ayuda al desarrollo y el papel relativo de las políticas internacionales en las políticas de desarrollo en el marco de acción global y multilateral.

De hecho, en términos generales, se desprende que los países que más deberían estar comprometidos no están cumpliendo con los compromisos de ayuda adquiridos. Así, no se debe obviar el esfuerzo económico que supone la consecución de los Objetivos de Milenio, que suponen los 195.000 millones de dólares al año (según estimaciones de las propias Naciones Unidas) lo que supone el 0,54\% del Producto Interior Bruto de los países desarrollados.

En esta línea, recientemente han aparecido dos ideas íntimamente vinculadas y que se configuran también como cruciales para lograr progresos en la consecución de los Objetivos que son la responsabilidad mutua y la gobernabilidad. Por tanto, el seguimiento del desempeño y de los progresos con respecto a estos compromisos es esencial para que todas las partes asuman la responsabilidad, haciendo hincapié en la necesidad de combatir el pernicioso fenómeno de la corrupción mediante la incorporación de manera permanente por parte de todos los agentes de mejoras en la gobernabilidad.

Como conclusión, puede afirmarse que se ha iniciado el camino pero queda aún un largo trayecto, el cual no está exento de dificultades. La globalización económica y financiera presenta, en el contexto socioeconómico, amplias ventajas que deben ser

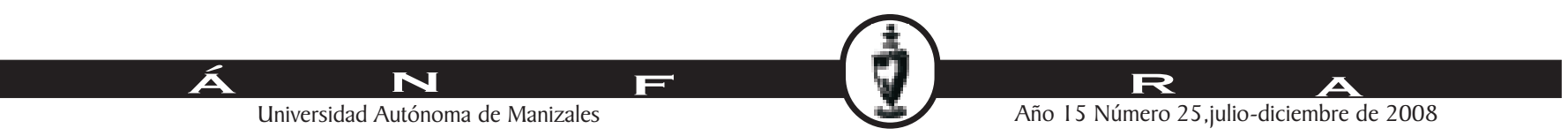


aprovechadas por las instituciones multilaterales de cooperación para conseguir sus propósitos principales. Los Objetivos del Milenio pueden (y deben) marcar un hito en este sentido pero, para ello, hay que tener presente de manera constante que se precisan más inversiones para realizar el seguimiento de algunos aspectos de la gestión de gobierno de los países. Entre estas cuestiones cabría destacar la administración de las finanzas públicas o las prácticas de adquisiciones que deben ser evaluadas y controladas de modo correcto y continuo. Así se conseguiría no sólo determinar los progresos logrados en la consecución de los Objetivos, sino también aspectos tan necesarios como generar mayores exigencias en cuanto a la gobernabilidad y aumentar la rendición de cuentas.

En definitiva, y tal como se recoge en la propia Declaración del Milenio (NN.UU, 2000), "el cambio de siglo constituye un momento único y que simbólicamente apremia a los 189 Estados Miembros para articular y afirmar una visión llena de inspiración para las Naciones Unidas en la nueva era". De nosotros, y nadie más, depende el ser capaces de conseguirlo.

\section{Bibliografia}

COMITÉ DE PROTECCIÓN SOCIAL; (2002). Lucha contra la pobreza y la exclusión social: objetivos comunes para la segunda ronda de los planes de acción nacionales Medidas de apoyo. Rev. 14/64/1/02; Consejo Europeo.

COMITÉ PARA EL DESARROLLO; (2006). Informe sobre seguimiento mundial 2006. INTENSIFICACIÓN DE LAS RESPONSABILIDADES MUTUAS: asistencia, comercio y gobernabilidad. Fondo Monetario Internacional y Banco Mundial, DC 2006-0004, 6 abril de 2006.

FORO DE ALTO NIVEL; (2005). Declaración de Paris sobre Eficacia de la Ayuda al Desarrollo. Apropiación, Armonización, Alineación \& Resultados y Mutua Responsabilidad. Paris, Francia.

LARRÚ, J.M. (2006). "La Ayuda al Desarrollo, ¿Reduce La Pobreza?" en VIII Reunión de Economía Mundial, Alicante, abril 2006

MEJ ÍA, H.; (2002). Discurso de apertura de la XII Cumbre I beroamericana de J efes de Estado y de Gobierno. Bávaro, República Dominicana.

NACIONES UNIDAS; (2000). Cumbre del Milenio de las Naciones Unidas; Resolución de IA ASAMBLEA GENERAL A/RES/54/254. NNUU, Nueva York, EEUU.

NACIONES UNIDAS; (2005). Objetivos de desarrollo del Milenio. Informe de 2005. Nueva York, EEUU.

ORGANIZACIÓN MUNDIAL DEL COMERCIO; (2001). Declaración Ministerial. Doha, EEUU.

PÉREZ DE LAS HERAS, M. (2002). La cumbre de Johannesburgo. Ediciones MundiPrensa, Madrid.

SACHS, J. D.S. (2002). "Los males de la globalización" en El País 10 de noviembre de 2002. Madrid, España.

WOLFENSOHN, J.D.; (2002). "El reto de Johanesburgo" en El País 23 de agosto de 2002. Madrid, España.

WOLFOWITZ, P.; (2006). Conferencia de prensa del Comité para el Desarrollo del FMI y el Banco Mundial. Press Release No:2006/376/EXC. Washinton, Estados Unidos.

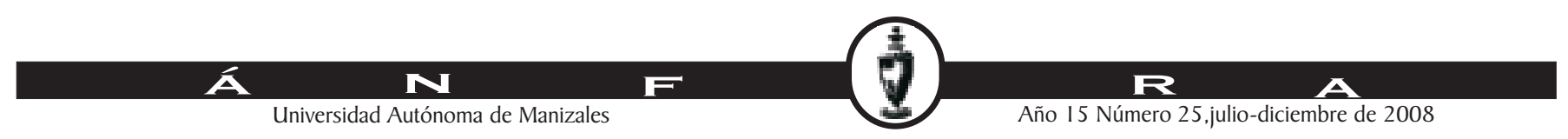

